\title{
Tratamento endovascular de pseudoaneurisma de artéria mesentérica superior: relato de caso
}

\author{
Endovascular treatment for superior mesenteric artery pseudoaneurysm: case report
}

Felipe Nasser', Breno Boueri Affonso², Seleno Glauber de Jesus-Silva ${ }^{3}$, Raimundo Teixeira de Araújo Júnior ${ }^{4}$, Mário Sérgio

Duarte Andrioli, Juliana Carvalho de Campos ${ }^{6}$, Rogério Carballo Afonso ${ }^{7}$, Ben-Hur Ferraz-Neto ${ }^{8}$

\begin{abstract}
Resumo
O pseudoaneurisma de artéria mesentérica superior é uma doença rara, porém com alta taxa de ruptura e mortalidade. Sua etiologia geralmente é infecciosa e comumente o diagnóstico é feito por meio de achado nos exames de imagem. Historicamente, seu tratamento de eleição tem sido o reparo cirúrgico aberto; entretanto, é associado a inúmeras complicações e dificuldades técnicas. Relatamos um caso de pseudoaneurisma de artéria mesentérica superior em um paciente portador de abscesso hepático no qual, após resolução do processo infeccioso, foi empregado, com sucesso, um tratamento minimamente invasivo endovascular, com implante de micromolas e stent não-recoberto.
\end{abstract}

Palavras-chave: Artéria mesentérica superior, falso aneurisma, embolização terapêutica.

\begin{abstract}
Pseudoaneurysm of the superior mesenteric artery is a rare disease, however it is associated to a high incidence of rupture and mortality. The etiology is usually infectious and the diagnosis is commonly made by means of occasional imaging. Historically, the treatment of choice has been open surgical repair; however it is associated to numerous complications and technical difficulties. We reported a case of a pseudoaneurysm of superior mesenteric artery in a patient with liver abscess who, after resolution of infection, underwent successfully a minimally invasive endovascular approach, with deployment of microcoils and bare stent.
\end{abstract}

Keywords: Mesenteric artery, superior, aneurysm, false, embolization, therapeutic.

Introdução

Os aneurismas e pseudoaneurismas de artérias viscerais são entidades raras, com prevalência na faixa de 0,1 a $2 \%$ da população em geral ${ }^{1}$. Apesar de o aneurisma verdadeiro da artéria mesentérica superior (AMS) ser raramente descrito na literatura, o pseudoaneurisma, considerado uma ruptura contida da parede arterial, é o quarto tipo mais comum dos aneurismas viscerais, com prevalência de $4 \%$. Possui altas taxas de ruptura (20 a $30 \%$ ) e de mortalidade (30 a 50\%). Alguns relatos recentes advogam o tratamento endovascular como opção elegante e minimamente invasiva para esses pacientes ${ }^{1,2}$. Descrevemos um caso de pseudoaneurisma de AMS tratado através de abordagem endovascular no Departamento de Radiologia Intervencionista do Hospital Israelita Albert Einstein (HIAE).

\section{Descrição do caso}

Paciente masculino, 53 anos, admitido com quadro abdominal infeccioso, com imagem ultrassonográfica

Trabalho realizado no Departamento de Radiologia Intervencionista do HIAE, São Paulo (SP), Brasil

1. Doutor; Chefe do Departamento de Radiologia Intervencionista do Hospital Israelita Albert Einstein (HIAE), São Paulo (SP), Brasil

2. Especialista em Radiologia Intervencionista e Cirurgia Endovascular (RICE); Radiologista Intervencionista do HIAE, São Paulo (SP), Brasil

3. Especialista em RICE; Radiologista Intervencionista do HIAE, São Paulo (SP), Brasil

4. Especialista em RICE; Estagiário do Departamento de Radiologia Intervencionista do HIAE, São Paulo (SP), Brasil

5. Neurointervencionista do HIAE, São Paulo (SP), Brasil

6. Biomédica do Departamento de Radiologia Intervencionista do HIAE, São Paulo (SP), Brasil

7. Mestre em Cirurgia; Cirurgião da Equipe de Transplante Hepático do HIAE, São Paulo (SP), Brasil

8. Livre-Docente em Cirurgia, Coordenador da Equipe de Transplante do HIAE

Não foram declarados conflitos de interesse associados à publicação deste artigo

Artigo submetido em 11.11.2009, aceito em 28.01.2010

J Vasc Bras. 2010;9(3):182-185 
sugestiva de abscesso hepático no lobo direito, entre os segmentos VI e VII, medindo 5,9 x 5,8 x 4,6 cm. Após drenagem percutânea do mesmo, uma amostra da secreção purulenta obtida foi encaminhada para cultura, porém não houve crescimento bacteriano. Após antibioticoterapia empírica, foi realizada tomografia computadorizada de abdome, que evidenciou pequena coleção residual de $1,8 \mathrm{x}$ $1,4 \mathrm{~cm}$ no segmento VII. Simultaneamente, foi detectada

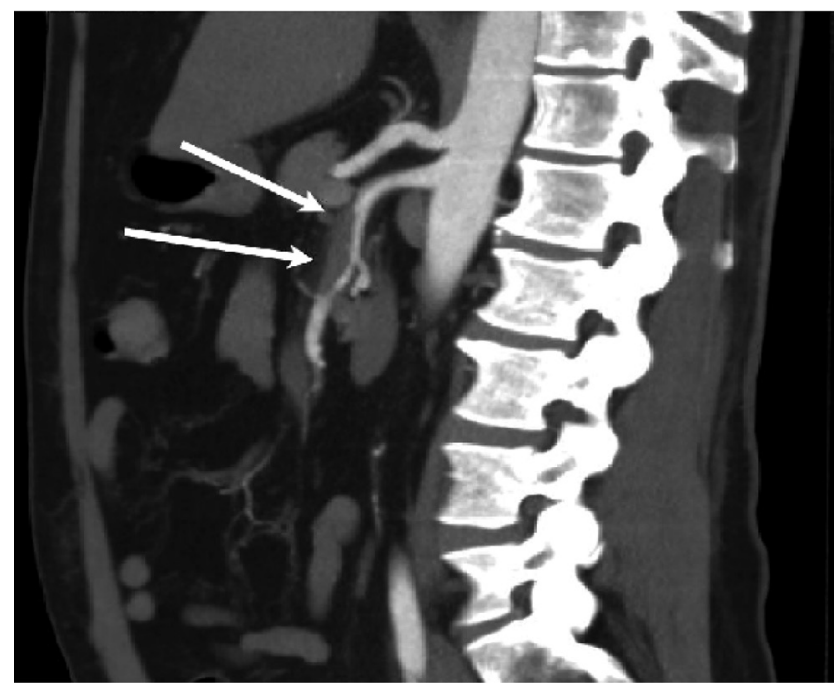

Figura 1 - Estudo angiotomográfico evidenciando pseudoaneurisma em artéria mesentérica superior com compressão da luz por trombo intramural

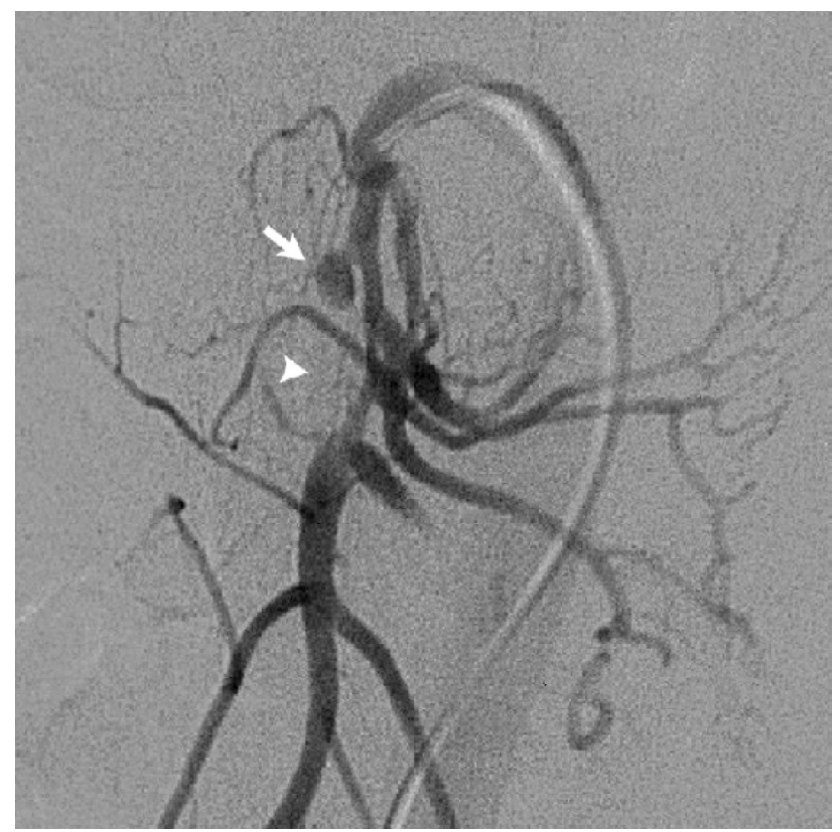

Figura 2 - Angiografia mesentérica superior evidenciando dilatação sacular em segmento proximal (seta) e compressão da luz arterial próximo à origem dos ramos jejunais (ponta da seta) uma dilatação aneurismática da porção proximal da AMS, com trombos luminais, determinando estenose moderada da mesma (Figura 1). Após melhora clínica, programouse angiografia juntamente com a proposta de tratamento endovascular.

O procedimento foi realizado sob anestesia geral e antiagregação plaquetária com clopidogrel 300 mg. Após punção femoral e cateterismo da AMS, o exame angiográfico mostrou dilatação aneurismática do tipo sacular em seu terço proximal, seguido de estenose grave e extensa na emergência dos ramos jejunais e lentificação do fluxo de contraste para ramos jejunoileais e cólicos, sendo feito o diagnóstico de pseudoaneurisma com compressão extrínseca da luz arterial (Figura 2). Foi então introduzido um cateter-guia $6 \mathrm{~F}$ até o óstio da AMS, seguido do cateterismo superseletivo do pseudoaneurisma com microcateter e da embolização do mesmo com micromolas de platina de liberação controlada. Efetuou-se, em seguida, a transposição da estenose, com o implante de stent de nitinol autoexpansível de $7 \mathrm{~mm}$ de diâmetro. O controle angiográfico demonstrou implante adequado do stent, bem como a preservação do tronco e ramos da AMS e melhora da perfusão das alças intestinais (Figura 3). O controle por angiotomografia após um mês mostrou perviedade da artéria mesentérica superior e exclusão do saco pseudoaneurismático (Figura 4).

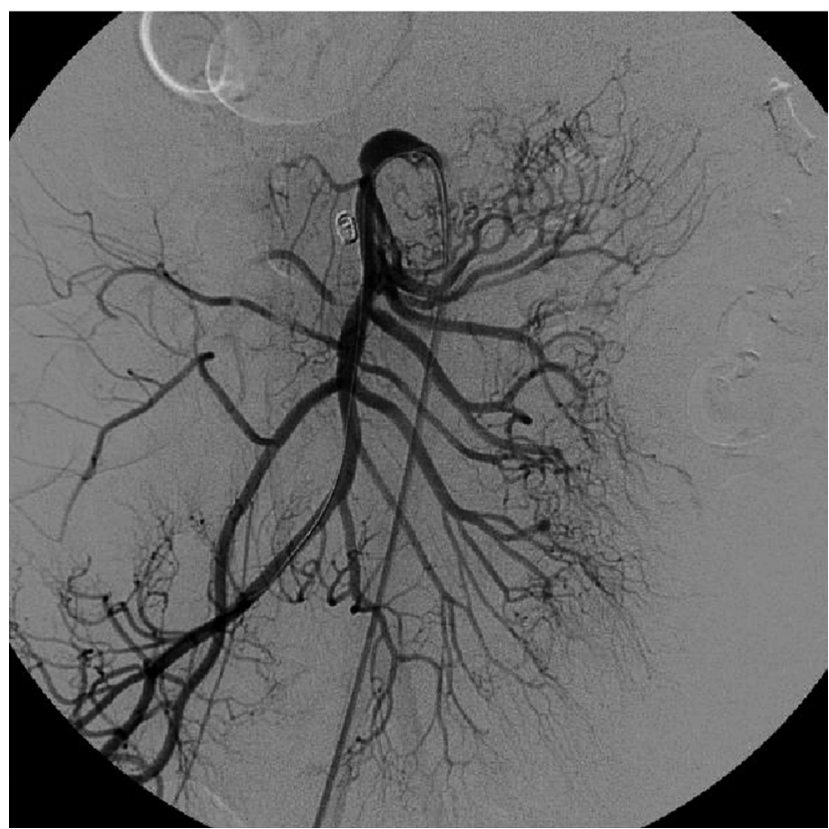

Figura 3 - Estudo angiográfico após tratamento evidenciando exclusão do pseudoaneurisma com presença de molas e melhora da perfusão dos ramos intestinais 


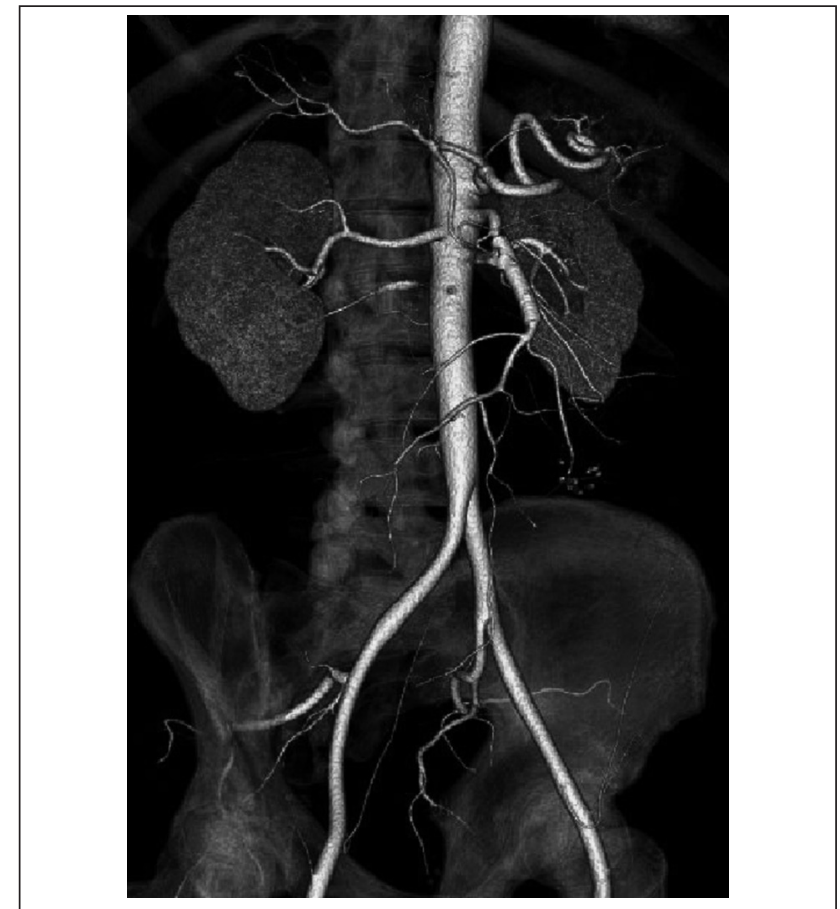

Figura 4 - Angiotomografia, controle de um mês pós-embolização, observando-se perviedade da artéria mesentérica superior

\section{Discussão}

O aneurisma da AMS foi descrito primeiramente por Koch em 1951. DeBakey e Cooley descreveram em 1953 o primeiro tratamento bem sucedido de um paciente com aneurisma de AMS, realizando a ressecção sem reconstrução arterial. Sua incidência é subestimada devido a sua localização, o que dificulta seu diagnóstico. A faixa etária mais acometida é entre 45 e 55 anos, com distribuição igual entre os sexos. Cerca de 58 a $63 \%$ dos pseudoaneurismas da AMS são infecciosos, dos quais os principais agentes etiológicos são os Streptococos ssp. e Stafilococos ssp, geralmente associados a quadros de endocardite e bacteremia ${ }^{3}$. Outros fatores etiológicos descritos são a aterosclerose, vasculites, fibrodisplasia, pancreatites e até mesmo a litotripsia renal ${ }^{4}$. Mitchell et al. descreveram a deficiência do complexo elastase/alfa-antitripsina como fator etiológico e Tupler et al., a associação com ergotismo ${ }^{5}$.

Os pseudoaneurismas da AMS são, na sua maioria, assintomáticos. Cerca de 40 a $80 \%$ dos casos evoluem para ruptura sem sinais e sintomas prodrômicos, mas 70 a $80 \%$ dos pacientes com ruptura são sintomáticos e têm elevada taxa de mortalidade. A dor aguda intensa com sinais e sintomas de choque hemorrágico é associada à ruptura. $\mathrm{O}$ exame físico é escasso devido à localização e ao tamanho do pseudoaneurisma, mas uma massa abdominal dolorosa e móvel pode ser observada em casos de ruptura ${ }^{6}$. A angiotomografia multislice vem se destacando no diagnóstico por ser um exame não-invasivo, podendo avaliar sua dimensão, sua relação com órgãos adjacentes e a presença de trombos intraluminais. A arteriografia deve ser reservada somente para o uso durante o tratamento endovascular.

Enquanto o reparo dos aneurismas viscerais verdadeiros é geralmente indicado somente naqueles com mais de 1,5 a $2 \mathrm{~cm}$ de diâmetro - ou nos casos sintomáticos, como os aneurismas rotos, e em pacientes do sexo feminino com idade fértil - considera-se que todos os casos de pseudoaneurismas devam ser tratados, independentemente do tamanho ${ }^{1,7}$.

O tratamento cirúrgico convencional é aceito ainda como modalidade terapêutica de escolha em pacientes de baixo risco. É realizada, em geral, a ligadura proximal e distal do aneurisma, seguida de reconstrução aortomesentérica com prótese $^{8}$. Possui a vantagem de ser um reparo definitivo e com baixo risco de re-estenose quando realizado por cirurgiões experientes. Suas desvantagens são o maior risco cirúrgico-anestésico, hemorragia e infecção, maior curva de aprendizado, difícil acesso operatório, risco de torções e oclusão dos enxertos.

Com o aprimoramento das técnicas endovasculares e dos dispositivos utilizados, o método tem se tornado para muitos a terapia de escolha, principalmente em pacientes de alto risco. Tem como principais vantagens a possibilidade de ser realizada sob anestesia local, além do controle angiográfico pós-procedimento imediato, menor risco de íleo paralítico, abscessos, hemorragias e recuperação mais rápida ${ }^{9}$. As modalidades da terapêutica endovascular são as embolizações com cianoacrilato (cola) ou molas (fibradas ou de platina) e a utilização de stents (revestidos ou não) ${ }^{10,11}$ e, em algumas ocasiões, sua associação ${ }^{7,12}$. As duas primeiras são utilizadas principalmente em aneurismas proximais, saculares e nos pseudoaneurismas. Os stents revestidos ou não-revestidos podem ser utilizados em quase todos os casos, mas principalmente quando se deseja manter a perviedade da artéria, e requerem condições anatômicas favoráveis para seu emprego ${ }^{13}$. Há relato também do uso de injeção de trombina dentro da luz do pseudoaneurisma, por meio de dispositivo de proteção embólica distal utilizado em intervenções carotídeas ${ }^{14}$.

As molas são dispositivos metálicos destinados a causar trombose permanente dos vasos. Podem ser de liberação controlada, permitindo um posicionamento mais preciso. Os stents vasculares compostos por liga metálica de níquel e titânio (nitinol) são os que têm melhor navegabilidade e 
maleabilidade, permitindo melhor acomodação na artériaalvo $^{15,16}$. O tratamento com stent exige condições anatômicas favoráveis para a realização do procedimento, tais como: colo proximal e distal, calibre adequado do vaso e pouca tortuosidade. Pacientes sem esses critérios são considerados de anatomia complexa, sendo encaminhados ao tratamento cirúrgico convencional. Mesmo assim, com a maior experiência dos radiologistas intervencionistas e melhoria dos materiais, muitos pacientes com anatomia mais complexa têm sido tratados adequadamente pelo método minimamente invasivo.

\section{Conclusão}

O tratamento endovascular é considerado atualmente como de escolha para os aneurismas viscerais, entre os quais o pseudoaneurisma de AMS. A constante evolução dos dispositivos endovasculares provavelmente permitirá o tratamento da maior parte dos pacientes, incluindo aqueles com anatomia desfavorável. O seguimento da perviedade em longo prazo é necessário para a avaliação da eficácia do método.

\section{Referências}

1. Zelenock G, Stanley J. Splanchnic artery aneurysms. In: Rutherford R, editor. Vascular surgery. 5th ed. Philadelphia: WB Saunders; 2000. p.1124-39.

2. Mattar SG, Lumsden AB. The management of splenic artery aneurysms: experience with 23 cases. Am J Surg. 1995;169:580-4.

3. Dziuban EJ, Teitelbaum DH, Bakhtyar A, et al. Mesenteric pseudoaneurysm and cerebral stroke as sequelae of infective endocarditis in an adolescent. J Pediatr Surg. 2008;43:1923-7.

4. Yan SL, Wu HS, Chou DA, et al. Pseudoaneurysm of superior mesentery artery branch after renal extracorporeal shock wave lithotripsy: case report and review. J Trauma. 2007;62:770-4.

5. Zimmerman-Klima PM, Wixon CL, Bogey WM Jr, Lalikos JF, Powell CS. Considerations in the management of aneurysms of the superior mesenteric artery. Ann Vasc Surg. 2000;14:410-4.

6. Abbas MA, Stone WM, Fowl RJ, et al. Splenic artery aneurysms: two decades experience at Mayo clinic. Ann Vasc Surg. 2002;16:442-9.

7. Tulsyan N, Kashyap VS, Greenberg RK, et al. The endovascular management of visceral artery aneurysms and pseudoaneurysms. J Vasc Surg. 2007;45:276-83.
8. Stone WM, Abbas MA, Cherry KJ, Fowl RJ, Gloviczki P. Superior mesenteric artery aneurysms: is presence an indication for intervention? J Vasc Surg. 2002;36:234-7.

9. Tessier DJ, Abbas MA, Fowl RJ, et al. Management of rare mesenteric arterial branch aneurysms. Ann Vasc Surg. 2002;16:586-0.

10. Yeo KK, Dawson DL, Brooks JL, Laird JR. Percutaneous treatment of a large superior mesenteric artery pseudoaneurysm and arteriovenous fistula: a case report. J Vasc Surg. 2008;48:730-4.

11. Suzuki K, Mori Y, Komada T, Matsushima M, Ota T, Naganawa $\mathrm{S}$. Stent-graft treatment for bleeding superior mesenteric artery pseudoaneurysm after pancreaticoduodenectomy. Cardiovasc Intervent Radiol. 2009;32:762-6.

12. Ozaki T, Kimura M, Yoshimura N, et al. Endovascular treatment of spontaneous isolated dissecting aneurysm of the superior mesenteric artery using stent-assisted coil embolization. Cardiovasc Intervent Radiol. 2006;29:435-7.

13. Tan M, Di Carlo A, Stein LA, Cantarovich M, Tchervenkov Jl, Metrakos P. Pseudoaneurism of the superior mesenteric artery after pancreas transplantation treated by endovascular stenting. Transplantation. 2001;72:336-8.

14. Juszkat R, Krasiński Z, Wykrętowicz M, Staniszewski R, Majewski W. Transarterial thrombin injection secured with an embolic protection device as a treatment for a superior mesenteric artery pseudoaneurysm. Cardiovasc Intervent Radiol. [revista eletrônica]. 2010 Jan 8. http://www.springerlink.com/content/dh61346v1uk01157/ fulltext.pdf.

15. Grego FG, Lepidi S, Ragazzi R, lurilli V, Stramanà R, Deriu GP. Visceral artery aneurysms: a single center experience. Cardiovasc Surg. 2003;11:19-25.

16. Carmeci C, McClenathan J. Visceral artery aneurysms as seen in a community hospital. Am J Surg. 2000;179:486-9.

Correspondência:
Felipe Nasser
Departamento de Radiologia Intervencionista do HIAE
Avenida Albert Einstein, 627/701 - Morumbi
CEP 05652-000 - São Paulo (SP), Brasil
Fone: (11) 2151-1233
E-mail: felipenasser@einstein.br
Contribuições dos autores
Concepção e desenho do estudo: BBA, BEFN, RCA e MSDA
Análise e interpretação dos dados: BBA e RTAJ
Coleta de dados: JCC
Redação do artigo: SGJS
Revisão crítica do texto: FN
Análise estatística: N/A
* Todos os autores leram e aprovaram a versão final submetida ao J Vasc Bras.

